\title{
Splay-to-Bend Transition-Free Reactive Monomer Modified Pi-Cell
}

\author{
Szu-Fen F. Chen, Huang-Ming P. Chen, Liansing Chow, Yu-Yun Chang, and Han-Ping D. Shieh, Member, IEEE
}

\begin{abstract}
A reactive monomer modified Pi-cell (RMM-Pi-cell) comprising a layer of liquid crystal (LC) reactive monomer on one surface was prepared to control the surface pretilt angle. The simulation results suggested that a transition-free Pi-cell can be prepared by asymmetrical cell with one $8^{\circ}$ pretilt angle and the other surface greater than $47^{\circ}$ when the cell gap was smaller than $4 \mu \mathrm{m}$. The nematic reactive monomer layer has a molecular average tilt angle over $80^{\circ}$ which allowed the $\mathrm{LC}$ molecules to be arranged in a favored bend state in the asymmetrical cell. The critical voltages cannot be found in all 3- $\mu \mathrm{m}$ RMM-Pi-cells. The cell retardation data confirmed the initial bend orientation with zero voltage applied. Moreover, the light leakage of dark state was reduced. The contrast ratio of RMM-Pi-cell was improved by a factor of 11 compared with an original $\mathrm{Pi}$-cell without using compensation film.
\end{abstract}

Index Terms-Display, liquid crystal device, liquid crystal display (LCD), liquid crystals (LCs).

\section{INTRODUCTION}

$\mathbf{P}$ I-CELL, also known as optically compensated bend mode liquid crystal (LC) cell [1], possesses the advantages of wide viewing angles and fast response time for liquid crystal display (LCD) application. To be useful, the conventional Pi-cells are challenged by two major issues: the splay-to-bend transition, for one, is inevitably present. Without applying a higher voltage, the active bend configuration relaxes into splay configuration [2]-[4]. The other drawback is the low contrast ratio caused by the light leakage in the dark state in which LC molecules cannot be vertically arranged under a strong boundary condition. Many research efforts have been intensively involved in solving the splay-to-bend transitional issue by using various methods: an electrical twist field in Pi-cell [5],

Manuscript received December 04, 2008; revised February 02, 2009. First published February 24, 2009; current version published April 29, 2009. This work was supported in part by the National Science Council of the Republic of China under Grant NSC96-2221-E-009-078-, in part by MOEA Technology Development for Academic Project 96-EC-17-A-07-S1-046, and in part by Chunghwa Picture Tubes, Ltd. (CPT).

S.-F. F. Chen was with the Department of Photonics and Electro-Optics Institute, National Chiao Tung University, Hsinchu, 30010 Taiwan. She is now with Panel Design Division, Central Research Institute, Small and Medium TFT Product BU, Chung-Hwa Picture Tubes, Ltd., Taoyuan 326, Taiwan (e-mail: fenny@mail.cptt.com.tw).

H.-M. P. Chen, L. Chow, Y.-Y. Chang are with the Department of Photonics and Display Institute, National Chiao Tung University, Hsinchu, 30010 Taiwan (e-mail: pchen@mail.nctu.edu.tw; liliane.ep91@nctu.edu.tw; clouding.di96g@nctu.edu.tw).

H.-P. D. Shieh is with the College of Electrical and Computer Engineering, Department of Photonics and Display Institute, National Chiao Tung University, Hsinchu, 30010 Taiwan (e-mail: hpshieh@mail.nctu.edu.tw).

Color versions of one or more of the figures in this letter are available online at http://ieeexplore.ieee.org.

Digital Object Identifier 10.1109/LPT.2009.2015771 (a)
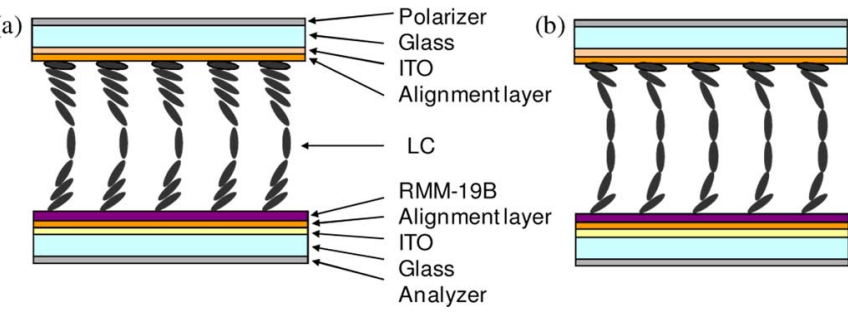

Fig. 1. Scheme of RMM-Pi-cell under (a) 0 and (b) $6 \mathrm{~V}$.

polymer-stabilized walls [6]-[8], increasing surface pretilted angle [4], [9], and multidomain alignment [9]-[11], etc. In this study, we report a transition-free reactive monomer modified Pi-cell (RMM-Pi-cell), in which the splay-to-bend transition is suppressed and contrast ratio is enhanced by a factor of 11 more than a conventional Pi-cell.

\section{EXPERIMENTS AND SIMULATIONS}

\section{A. RMM-Pi-Cell Fabrication}

The polyimide (PI) alignment film (PIA-5580-01, Chisso Corporation) was prepared by spin-coating on a $2 \mathrm{~cm} \times 2.5 \mathrm{~cm}$ indium-tin-oxide (ITO) glass and hard baked at $220{ }^{\circ} \mathrm{C}$ for $1 \mathrm{~h}$. The $8^{\circ}-12^{\circ}$ pretilt angles were obtained after buffing the cured PI film. The propylene glycol monomethyl ether acetate solutions, comprising various concentrations of reactive monomer (RMM-19B, Merck Chemicals Ltd.), were spin-coated on a PI buffed ITO glass. The thickness of reactive monomer (RM) film was controlled by the solution's concentration and the spin coater's speed. The film thickness was measured by the height of the cross section, using atomic force microscope. RMM-Pi-cell was assembled with RM on one side and buffed PI surface on the other side, as shown in Fig. 1. The cell gap was controlled at either 3.3 or $4.7 \mu \mathrm{m}$. The LC (ZCE-5096XX, Chisso Corporation) was filled by capillary force into the cell for electrooptical characterizations. The retardations of RMM-Pi-cells and RM thin films were measured by a Soleil-Babinet compensator.

\section{B. Critical Pretilt Angle Estimation}

In order to verify the initial bend orientation without splay configuration in the RMM-Pi-cell, we calculated the lowest pretilt angle requirements and probed the RM's surface pretilt angle, in addition to the cell retardation's measurement. In the calculation [4], the symmetrical Pi-cell was considered first; the relationship between the driving voltage and electric field 
can be simplified as $E \sim U / d$. The Gibbs free energy of spay and bend cells can be calculated by [12], [13]

$$
\begin{aligned}
G=\frac{1}{2} \int_{0}^{d}\left[\left(K_{11} \cos ^{2} \theta+K_{33} \sin ^{2} \theta\right)\right. & \left(\frac{d \theta}{d z}\right)^{2} \\
& \left.-\varepsilon_{0} \Delta \varepsilon E^{2} \sin ^{2} \theta\right] d z
\end{aligned}
$$

where $K_{11}$ and $K_{33}$ denote the splay and bend elastic constants, and $\varepsilon_{0}$ and $\Delta \varepsilon$ are the dielectric constant in vacuum and dielectric anisotropy, respectively. $\theta(z)$ is the LC tilt angle in the $z$-direction perpendicular to the glass substrate. The critical pretilt angle $\alpha_{c}$ for a transition-free Pi-cell can be derived from [12], [14]

$$
\left(K_{33}-K_{11}\right) \sin \left(2 \alpha_{c}\right)+\left(K_{33}+K_{11}\right)\left(\pi-4 \alpha_{c}\right)=0 .
$$

Based on the calculation, the critical pretilt angle is around $46^{\circ}$ for a symmetrical Pi-cell to form a bend state configuration (LC: ZCE-5096XX, $K_{11}=9.8 \mathrm{pN}, K_{33}=11.8 \mathrm{pN}$ at $20{ }^{\circ} \mathrm{C}$ ). By varying the surface's pretilt angle on both top and bottom surfaces for asymmetrical cells, the voltage versus transmittance $(V-T)$ curves simulated by DIMOS 2.0-Display Modeling System (autronic-MELCHERS GmbH) suggested that the bend state configuration can also be achieved by surface pretilt angle of top/bottom substrates at $8^{\circ} / 47^{\circ}$ in a $4-\mu \mathrm{m}$ cell.

It is due to the Gibbs energy of the bend state configuration $\left(6.6 \mu \cdot \mathrm{J} / \mathrm{m}^{2}\right)$ lower than the spray state. The simulation result suggested that a transition-free Pi-cell can be prepared by an asymmetrical cell with one regular $8^{\circ}$ pretilt angle and the other surface greater than $47^{\circ}$ when its cell gap is smaller than $4 \mu \mathrm{m}$.

\section{RESUlTS AND DISCUSSION}

The critical voltages to hold the bend state measured at $\lambda=$ $633 \mathrm{~nm}$ were $0.6,0.2$, and $0.0 \mathrm{~V}$ for RM's thickness of 80 , 110 , and $134 \mathrm{~nm}$ in 4.7- $\mu \mathrm{m}$ RMM-Pi-cells, respectively. On the contrary, the splay-to-bend transition was not found even in the thin RM layer for all 3.3- $\mu$ m cells. The experimental results confirmed the DIMOS simulation. Further investigation was carried out to determine the RM's surface pretilt angle. RMM-19B's $\Delta n_{\mathrm{RM}}$ (at $\lambda=452 \mathrm{~nm}$ ), 0.1449, was obtained from a $1.58-\mu \mathrm{m}$ homogenous planar cell. Based on the measured result $\left(\Delta n_{\mathrm{RM}}=0.1449\right)$, the retardation of a $200-\mathrm{nm}$ homogenous RM thin film was at $28.98 \mathrm{~nm}$. The thin RM film's retardations, however, were observed at $0.17,0.09$, and $0.11 \mathrm{~nm}$ for the thickness of 237, 120, and $100 \mathrm{~nm}$, respectively. The discrepancy suggested that the liquid crystalline molecular orientation in the RM film was highly tilted. In order to simplify the LC's pretilt angle calculation, we assumed a uniform molecular tilt orientation for RMM-19B film. Substituting the retardation values into (3), the average tilt angles $\theta$ of RM layers were calculated at $84.5^{\circ}$ to $86^{\circ}$ with $n_{o}=1.5$, according to various film
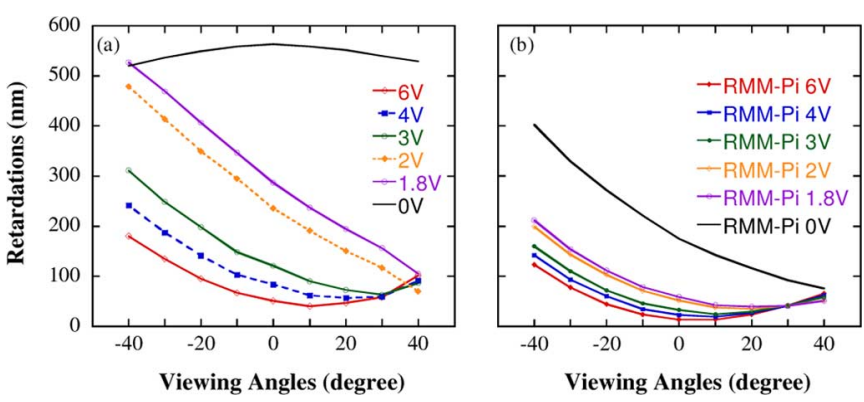

Fig. 2. Retardations of (a) the conventional Pi-cell, and (b) RMM-Pi-cells with different driving voltage from 0 to $6 \mathrm{~V}$ in different viewing angles.
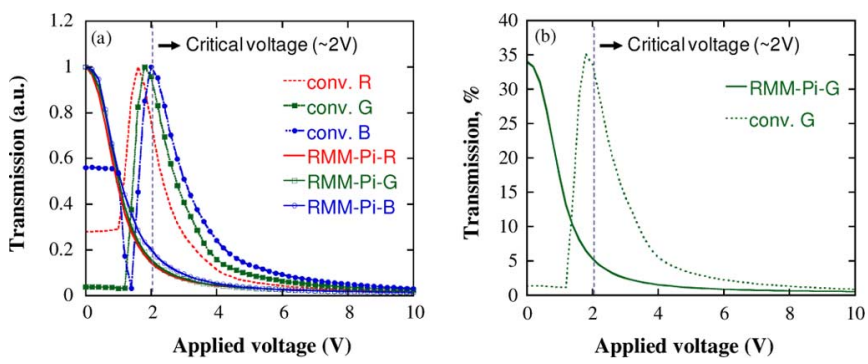

Fig. 3. (a) Normalized RGB's $V-T$ curve, and (b) unnormalized green $V-T$ curve of RMM-Pi-cell and conventional Pi-cell without compensation films.

thickness. The deviation of tilt angle was around $\pm 0.02^{\circ}$ when the RM's $n_{o}$ increased or decreased by $10 \%$

$$
\frac{1}{n_{e-\mathrm{RM}}^{2}(\theta)}=\frac{\sin ^{2} \theta}{n_{o-\mathrm{RM}}^{2}}+\frac{\cos ^{2} \theta}{n_{e-\mathrm{RM}}^{2}}
$$

where

$$
n_{e-\mathrm{RM}}(\theta)=\Delta n_{\mathrm{RM}}(\theta)+n_{o-\mathrm{RM}} .
$$

The calculated average RM tilt angle is much greater than the theoretical critical pretilt angle required in an asymmetrical $\mathrm{Pi}$-cell. As a result, the LC molecules in RMM-Pi-cell favored in bend orientation at its initial state without applying voltage [15]. To further confirm the bend state orientation, the cell retardations of both conventional Pi-cell and RMM-Pi-cell were measured by applying different driving voltages. As shown in Fig. 2, the conventional Pi-cell has larger retardation curves compared with the RMM-Pi-cell, when the applying voltage is higher than the critical voltage. This result confirmed that the LC molecules were arranged in the bend orientation at its initial state without applying any voltage in the RMM-Pi-cell.

The normalized $V-T$ curves of RMM-Pi-cell shifting to the left without critical voltage confirmed that it was a transitionfree cell, as illustrated in Fig. 3(a). Furthermore, the unnormalized green $V-T$ curve indicated the transmittance in bright state was not seriously compromised in the RMM-Pi-cell, as shown in Fig. 3(b). The residual retardation from RM's splay orientation may have contributed to noncompromised transmittance in the RMM-Pi-cell compared to the high tilted conventional Pi-cell. The light leakage after $4 \mathrm{~V}$ was largely suppressed in this cell structure.

The retardations of the RMM-Pi-cell corresponding to different viewing angles were lower than the conventional 


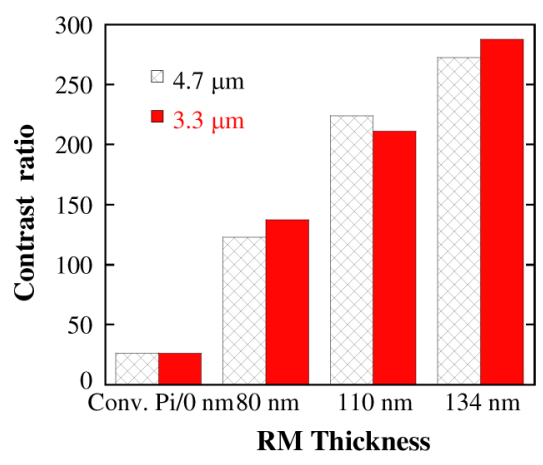

Fig. 4. Contrast ratio of RMM-Pi-cells in 4.7 and $3.3 \mathrm{~mm}$ without compensation films.

Pi-cell. The results suggested that the LC molecules of the RMM-Pi-cells can be arranged vertically, much closer to the cell boundaries at the dark state. The light leakage, therefore, was reduced in the RMM-Pi-cell. Without utilizing compensation films, the contrast ratio of the RMM-Pi-cell can be improved by a factor of 11 more than the conventional Pi-cell. The improved contrast ratios of RMM-Pi-cell cells in 4.7 and $3.3 \mu \mathrm{m}$ were shown in Fig. 4. The RMM-Pi-cell's driving voltage can be reduced to $0-6 \mathrm{~V}$ without applying a high voltage pulse. The viewing angle of RMM-Pi-cell was $40^{\circ} / 50^{\circ} / 60^{\circ} / 50^{\circ}$ with respect to $0^{\circ} / 90^{\circ} / 180^{\circ} / 270^{\circ}$ orientations without compensation film. The advantages of the transition-free RMM-Pi-cell are not only higher in contrast ratio, but also lower in power consumption than the conventional Pi-cell.

\section{CONCLUSION}

In summary, the RMM-Pi-cell can be driven without splay-tobend transition. The tilt angle of the RMM-Pi-cell has been confirmed theoretically and experimentally. The residual retardation of the splay-type thin film with a highly tilted surface angle maintained the bright state's transmittance and reduced the dark state's light leakage. The contrast ratio was improved up to a factor of 11 without using compensation films. Therefore, the proposed RMM-Pi-cell is suitable for low power consumption, for a better image quality in large size TFT-LCD applications.

\section{ACKNOWLEDGMENT}

H.-M. P. Chen would like to thank Chisso Corporation for providing the PIA-5580-01 polyimide.

\section{REFERENCES}

[1] Y. Yamaguchi, T. Miyashita, and T. Uchida, "Wide-viewing-angle display mode for the active-matrix LCD using bend-alignment liquid-crystal cell," in Soc. Inform. Disp. Symp. Dig., 1993, vol. 24, pp. 277-280.

[2] T. Satake and T. Kurata, Fast-Switching LCD With Dual-Domain Bend Mode Electronic Information Displays, IEICE Tech. Rep. 687, 2004, vol. 104, p. 9.

[3] X. D. Mi, M. Xu, D. K. Yang, and P. J. Bos, "Effects of pretilt angle on electro-optical properties of $\pi$-cell LCDs," in Soc. Inform. Disp. Symp. Dig., 1999, vol. 30, no. 5-1, pp. 1-4.

[4] F. S. Yeung and H. S. Kwok, "Fast-response no-bias-bend liquid crystal displays using nanostructured surfaces," Appl. Phys. Lett., vol. 88, p. $063505,2006$.

[5] K. Nakao, D. Suzuki, T. Kojima, M. Tsukane, and H. Wakemoto, "High-speed bend transition method using electrical twist field in OCB mode TFT-LCDs," in Soc. Inform. Disp. Symp. Dig., 2004, vol. 35, pp. 1416-1419.

[6] H. Kikuchi, H. Yamamoto, H. Sato, M. Kawakita, K. Takizawa, and H. Fujikake, "Bend-mode liquid crystal cells stabilized by aligned polymer walls," Jpn. J. Appl. Phys., vol. 44, pp. 981-989, 2005.

[7] S. H. Kim and L. C. Chien, "Electro-optical characteristics and morphology of a bend nematic liquid crystal device having templated polymer fibrils," Jpn. J. Appl. Phys., vol. 43, pp. 7643-7647, 2004.

[8] B.-R. Yang, S. J. Elston, P. Raynes, and H.-P. D. Shieh, "High-brightness relaxed-bend state in a Pi cell stabilized by synchronized polymerization," Appl. Phys. Lett., vol. 92, p. 221109, 2008.

[9] N. Nagae, T. Miyashita, T. Uchida, Y. Yamada, and Y. Ishii, "A novel method for high speed transition from splay to bend alignment in the OCB-mode LCD with fast response," in Soc. Inform. Disp. Symp. Digest., 2000, vol. 31, pp. 26-29.

[10] S. H. Lee, T. J. Kim, G. D. Lee, T. H. Yoon, and J. C. Kim, "Geometric structure for the uniform splay-to-bend transition in a_-cell," Jpn. J. Appl. Phys., vol. 42, pp. L1148-L1151, 2003.

[11] E. Acosta, B. Henley, D. Kean, M. Tillin, C. Tombling, M. Towler, E. Walton, H. Walton, and R. Winlow, "Nucleation of the Pi-cell operating state: A comparison of techniques," Liq. Cryst., vol. 31, pp. 1619-1625, 2004.

[12] F. S. Yeung, Y. W. Li, and H. S. Kwok, "Pi-cell liquid crystal displays at arbitrary pretilt angles," Appl. Phys. Lett., vol. 88, p. 041108, 2006.

[13] X. J. Yu and H. S. Kwok, "Bistable bend-splay liquid crystal display," Appl. Phys. Lett., vol. 85, p. 3711, 2004.

[14] Y. Sun, H. Ma, Z. Li, and Z. Zhang, "Pretilt angle effects on critical voltage and dynamic response of pi cell," Appl. Phys. Lett., vol. 90, p. 091103, 2007.

[15] C.-H. Chiu, H.-L. Kuo, P.-C. Chen, C.-H. Wen, Y.-C. Liu, and H. M. P. Chen, "Nanoimprinting-lithography-induced self-aligned liquid crystals for novel multifunctional optical films," Appl. Phys. Lett., vol. 88, p. $073509,2006$. 